\title{
Fatty acid composition in cerebral cortex, hippocampus and cerebellum in adult rats receiving salmon oil for 6 months
}

\author{
Maciej Firląg', Maciej Kamaszewski², Piotr Ostaszewski', Bożena Balasinska' \\ ${ }^{1}$ Department of Physiological Science, Faculty of Veterinary Medicine, Warsaw University of Life Science \\ ${ }^{2}$ Department of Ichthyobiology and Fisheries, Faculty of Animal Science, Warsaw University of Life Sciences
}

Firląg M, Kamaszewski M, Ostaszewski P, Balasinska B. Fatty acid composition in cerebral cortex, hippocampus and cerebellum in adult rats receiving salmon oil for 6 months. J Pre-Clin Clin Res. 2014; 8(1): 30-33.

\section{Abstract}

Introduction. Polyunsaturated fatty acids (PUFA) play an important role in the functioning of the brain, particularly in childhood and old age. The diet of pregnant mothers as well as very young children, affects the composition of fatty acids in childrens' brains and suggests the question of whether a diet enriched in n-3 PUFA throughout adult life may result in elevated levels of PUFA $n-3$ in individual brain regions, and thus delay the aging process.

Objective. The purpose of this research was to establish the fatty acid composition in the cerebral cortex, hippocampus and cerebellum in adult rats receiving for 6 months salmon oil, which is a rich source of n-3 fatty acid.

Materials and method. 6 -week-old rats were acclimatized for 2 weeks and then assigned into 2 groups ( $n=10)$ : control and fish oil supplemented. Both control and supplemented animals were fed a semi-synthetic, isoenergetic diet that varied only in fat composition. The brain fatty acids profile was determined by gas-chromatography analysis.

Results. Rats fed the supplemented diet showed an increased level of 22:6n-3 and a decreased level of 20:4n-6 and C22:5n-6 in all brain regions when compared to the control group. Furthermore, there was an increase of all monounsaturated fatty acids (MUFA) only in the hippocampus of supplemented rats when compared to the control group. Saturated fatty acids (SFA) content was only slightly modified in the administered diets.

Conclusions. A diet rich fish oil can restore an adequate level of fatty acid in the aging brain.

\section{Key words}

brain, fish oil, PUFA, aging

\section{INTRODUCTION}

A properly balanced diet that includes essential nutrients and delivers the right amount of energy has a significant impact on human health. It has been proved that a diet rich in certain biological components may protect organisms against certain diseases. An excellent example of this is the well-known Mediterranean diet and healthy eating habits in Japan. Those diets play a large part in better health due to their composition of active ingredients, but considerable interest is predicted in $n-3$ polyunsaturated fatty acids (PUFA), which are derived directly from vegetable oils and oily fish. Dietary fish oil treatment in humans has also been described in the diet of the Eskimo population [1]. The rarity of ischemic heart in Eskimo populations has been described and is believed to be related to the effect of the marinebased oils enriched in n-3 PUFA that were consumed. Early investigations also underscored the beneficial effects of PUFA in other illnesses, e.g., hypertension, gastrointestinal diseases, renal disease, arthritis, inflammatory processes, carcinomatosis, and diabetes mellitus [2]. It has also been shown that diet can affect the proper functioning of the brain. This beneficial role of $n-3$ PUFA has been observed for neurologic development and age-related impairments $[3,4]$. It has been reported that early dietary supply of n-3 PUFA improves later cognitive development in human infants and

Address for correspondence: mgr Maciej Firląg, Department of Physiological Science, Faculty of Veterinary Medicine, Warsaw University of Life Science-SGGW, 02-776 Warszawa, ul. Nowoursynowska 159, Poland e-mail: maciejfirlag@wp.pl

Received: 11 February 2014; accepted: 15 April 2014 memory-related learning ability in young rats. Other studies have shown the positive effects of n-3 PUFA supplementation in mood disorders, peroxisomal disorders, Parkinson's and Alzheimer's diseases [5, 6, 7].

One of the brain structures associated with learning and memory, as well as mood, is the hippocampus. Furthermore, the hippocampus is one of the regions of the adult brain where neurogenesis, which is the formation of new neurons, occurs. The rate of neurogenesis and survival of new neurons in adults is enhanced by many factors. This raises the question whether modulation of neurogenesis through proper diet could be a possible mechanism by which nutrition impacts on mental health. The aging of the brain and the weakening of control mechanisms may result in increased oxidative stress, loss of hippocampal volume, and an increase in markers of apoptosis observed in the cerebellum of rats. Cerebellar neurons are involved in modulating the force and range of movement and in the learning of motor skills. Cerebellum-dependent learning is associated with the Purkinje neuron number and is impaired by age-related decrements in morphology and function. Hippocampusdependent learning is associated with reduced capacity for new learning in pyramidal neurons in the perforant pathway in normal aging. Data about the adult life span in humans suggest that cerebellum-essential tasks show age-related deficits at earlier ages than hippocampus- essential tasks [8]. Many of the changes associated with aging are also described in the brain cortex. The cerebral cortex and hippocampus are severely affected by Alzheimer's disease. There is an urgent need for effective, mild therapy to prevent, delay or cure these disorders. 
Today, Western populations living both in urban and rural areas are experiencing increased life expectancy. The average index of life expectancy in Poland in 2009 reached the level of 80.2 years for women and 70.6 years for men. Concurrent with increases in life expectancy, a growing number of people suffer from neurodegenerative diseases. If the $n-3$ fatty acids support the treatment of neurodegenerative illnesses, then perhaps they may also help prevent premature aging of the brain. However, there is no conclusive data that would demonstrate whether and in which regions of the brain fatty acid composition is changed as a result of eating a diet rich in n-3 fatty acids for a long period of time. Because the greatest age-related changes in the brain occur in young and old individuals (decrease of PUFA level) it would be interesting to examine whether a diet enriched in n-3 PUFA throughout adult life may result in elevated levels of PUFA n-3 in individual brain regions, and thus delay the process of aging. Therefore, the purpose of this research was to establish the fatty acid composition in different brain structures (cerebral cortex, hippocampus and cerebellum) in adult rats receiving for 6 months salmon oil, which is a rich source of n-3 fatty acid.

\section{MATERIALS AND METHOD}

Animals and diets. The study design and experimental procedures were approved by the Animal and Ethics Review Committee at the University of Life Science in Warsaw (86/2010 III LKE). The animals were kept on a $12 \mathrm{~h}$ lightdark cycle. Room temperature was maintained at $22^{\circ} \mathrm{C}$, with average relative humidity at $70 \%$. Food and water were provided ad libitum. Body weight and food intake were measured once a week. Twelve Sprague-Dawley rats (Charles River Laboratories UK) were used in this study. The 6-weekold animals were acclimatized for 2 weeks and then assigned into 2 groups $(n=10)$ : control and fish oil supplemented. Both control and supplemented animals were fed a semi-synthetic, isoenergetic diet as previously described [4], which varied only in fat composition. The supplemented diet contained rapeseed oil $(20 \mathrm{~g} / \mathrm{kg})$, lard $(20 \mathrm{~g} / \mathrm{kg})$ and fish fat derived from salmon $(60 \mathrm{~g} / \mathrm{kg})$. The control diet lipids were a mixture of rapeseed oil $(10 \mathrm{~g} / \mathrm{kg})$ and lard $(90 \mathrm{~g} / \mathrm{kg})$. The fatty acid concentrations (\% of total fatty acids) in both diets are shown in Table 1. The supplemented diet contained 3.29\% EPA and 3.81\% DHA from fish fat, but the important source of $n-3$ fatty acids constituting 18:3n-3 originated from rapeseed oil. After 6 months, the rats were euthanized by intraperitoneal injection of sodium pentobarbital. Cerebral cortex, hippocampus and cerebellum were dissected and stored at $-80^{\circ} \mathrm{C}$ until further use.

Fatty acids analysis. Total lipids in diets and brain regions were extracted using a chloroform-methanol mixture $(2: 1, v / v)$, according to the Folch method [9]. Briefy, a sample of diet (1g) or brain homogenates $(200 \mathrm{mg}$ ) was extracted after homogenization and converted to fatty acids methyl esters (FAME). FAME were separated on a $100 \mathrm{~m} \times 0.25 \mathrm{~mm}$ capillary column filled with $0.2 \mu \mathrm{m} \mathrm{CP-Sil} 88$ stationary phase gas chromatograph equipped with autosampler and FID detector. The column temperature was kept at $170^{\circ} \mathrm{C}$ for the first $42 \mathrm{~min}$, raised to $240^{\circ} \mathrm{C}$ at the rate of $5^{\circ} \mathrm{C} / \mathrm{min}$, and finally maintained at this temperature for $31 \mathrm{~min}$. Identification of peaks corresponding to FAME was accomplished by means of a standard mixture of 37 FAMEs (Supelco, Belafonte,
Table 1. Fatty acid composition (\% total fatty acids) of the experimental diets

\begin{tabular}{|c|c|c|}
\hline Fatty acid & CON & SUP \\
\hline C12:0 & 1.63 & 2.79 \\
\hline C14:0 & nd & 0.2 \\
\hline C16:0 & 26.31 & 18.33 \\
\hline C18:0 & 35.18 & 24.3 \\
\hline$C 20: 0$ & 0.22 & 0.27 \\
\hline C22:0 & nd & 0.1 \\
\hline $\mathrm{C} 24: 0$ & nd & 0.6 \\
\hline$\Sigma$ SFA & 63.34 & 46.59 \\
\hline $\mathrm{C} 16: 1 \mathrm{n} 7$ & 2.2 & 2.71 \\
\hline C18:1n9 & 14.88 & 6.22 \\
\hline C18:1 n7 & 5.24 & 5.56 \\
\hline C20:1n9 & 1.42 & 1.67 \\
\hline C22:1n9 & nd & 2.69 \\
\hline$\Sigma$ MUFA & 23.74 & 18.85 \\
\hline C18:2n6 trans-LA & 2.64 & 3.45 \\
\hline C18:2n6 cis-LA & 8.6 & 13.2 \\
\hline$C 20: 3 n 6$ & 0.28 & 0.57 \\
\hline $\mathrm{C} 20: 4 \mathrm{n} 6 \mathrm{AA}$ & nd & 0.66 \\
\hline C22:5n6 DPA (n-6) & nd & 0.26 \\
\hline C18:3n3 ALA & 1.01 & 8.2 \\
\hline C20:5n3 EPA & nd & 3.29 \\
\hline C22:5n3 DPA (n-3) & nd & 0.14 \\
\hline $\mathrm{C} 22: 6 \mathrm{n} 3 \mathrm{DHA}$ & nd & 3.81 \\
\hline$\Sigma$ PUFA (n-6) & 11.52 & 18.14 \\
\hline$\Sigma$ PUFA (n-3) & 1.01 & 15.44 \\
\hline$\Sigma$ PUFA $(n-6+n-3)$ & 12.53 & 33.58 \\
\hline
\end{tabular}

CON - control diet, SUP - supplemented diet, SFA - saturated fatty acids, MUFA monounsaturated fatty acids, PUFA - polyunsaturated fatty acids, nd - not detected.

USA). The relative concentration of individual fatty acids was expressed as a percent of total fatty acids.

Statistical analysis. Data are expressed as means \pm standard deviation. Statistical analysis was performed by t-Student test. A p-value $<0.05$ was considered to be significant.

\section{RESULTS}

Feeding with the control and supplemented diet did not affect body weight between animals from each group (Tab. 2). Young rats (6-weeks-old) weighed $100 \pm 30 \mathrm{~g}$, and 6 months later, animals from the control and supplemented groups weighed $617.87 \pm 55.10$ and $620.55 \pm 51.99 \mathrm{~g}$, respectively. Brain weights did not differ between the groups.

The fatty acid composition varied according to the brain region, but the pattern of changes in PUFA and monounsaturated fatty acids (MUFA) levels in response to dietary treatments was relatively similar. On the other hand, the percentage change in the content of saturated fatty acids (SFA) was different, both between tissues and diets, without obvious trend. The saturated fatty acid (SFA) profile of dietary groups is given in Table 3 . The most significant change in the SFA content was found in the hippocampus. In the supplemented group, a decreased level was observed of hippocampal 14:0 (2.48 \pm 0.44$), 16: 0(30,12 \pm 0.59), 18: 0$ $(20.81 \pm 0.16)$ and an increased level of 24:0 (1.23 \pm 0.43$)$ fatty 
Table 2. Body and brain tissue weights of examined rats after 6 months of nutritional experiment

\begin{tabular}{lcc}
\hline \multicolumn{1}{c}{ Parameters } & CON & SUP \\
\hline Initial body weight $(\mathrm{g})$ & $100 \pm 30$ & $100 \pm 30$ \\
\hline Final body weight $(\mathrm{g})$ & $617.87 \pm 55.10$ & $620.55 \pm 51.99$ \\
\hline Brain $(\mathrm{g})$ & $2.09 \pm 0.1$ & $2.09 \pm 0.1$ \\
\hline Cerebral cortex $(\mathrm{g})$ & $0.64 \pm 0.04$ & $0.64 \pm 0.05$ \\
\hline Hippocampus $(\mathrm{g})$ & $0.12 \pm 0.02$ & $0.12 \pm 0.02$ \\
\hline Cerebellum $(\mathrm{g})$ & $0.33 \pm 0.01$ & $0.31 \pm 0.20$ \\
\hline
\end{tabular}

Values are mean \pm SD; CON - control diet, SUP - supplemented diet

acids when compared with control animals. In the cerebellum of the group fed the fish oil diet, a lower level was noted of 18:0 $(20.51 \pm 0.32)$ and an increased level of 24:0 $(1.39 \pm 0.87)$ fatty acids, and similarly in the hippocampus. The content of 14:0 and 16:0 in cerebellum did not change. In the cerebral cortex, the only statistically significant change was observed in the case of 24:0 fatty acids, which was lower in the supplemented rats (1.24 \pm 0.17$)$. compared to the control group $(2.25 \pm 0.26)$.

The level of MUFA also differed among brain structures. In general, there was an increase of the MUFA content in the supplemented rats when compared to the control group. In the hippocampus of animals fed fish oil, an increased level was noted of all analyzed MUFA. There was similar trend in the supplemented group in the cerebellum, except for 16:1n7 and in the cerebral cortex, except for $16: \ln 7$ and $18: \ln 9$, when compared to the control rats.

The fish oil diet substantially altered the PUFA profile in the examined brain regions with the exception of 18:2n- 6 and $22: 5 n-3$ in the hippocampus. Dietary supplemented animals had significantly higher $22: 6 \mathrm{n}-3$ in the cerebral cortex (23.01 \pm 0.52$)$, the hippocampus $(13.67 \pm 0.33)$ and the cerebellum (16.68 \pm 0.21$)$, compared with control groups. The levels of 22:6n-3 were also different in all brain regions in the control group, and the highest content of this fatty acid was observed in the cerebral cortex $(20.46 \pm 0.28)$; the lowest in the hippocampus $(12.47 \pm 0.34)$. The control groups tended to have significantly higher levels of 22:5n-6 in all tested tissues. The level of 22:5n-6 was highest in the cerebellum of control rats ( $4.17 \pm 0.61)$, less in the hippocampus $(3.45 \pm 0.28)$, and the lowest level was observed in the cerebral cortex $(3.28 \pm 0.26)$. In the supplemented group, significantly lower percentages of 22:5n-6 were found in all brain regions. In the case of $20: 4 n-6$, a similar trend was observed: fish oil supplementation reduces this fatty acid in the cerebral cortex (8.14 \pm 0.40$)$, the hippocampus $(8.73 \pm 0.22)$ and the cerebellum (5.43 \pm 0.21$)$, when compared to control group. The statistically important effect of the supplemented diet was also observed in the cerebral cortex and the cerebellum when the content of $18: 2 n-6$ and $22: 5 n-3$ was analysed. In the hippocampus, no changes were observed in these fatty acids.

\section{DISCUSSION}

Nutrition is an environmental variable that can affect the development of the brain during the period of growth, neurogenesis, synaptogenesis and maturation of neuronal pathways. The presented study aimed to determine to what extent fish oil supplementation affects the fatty acid lipid molecular profile in the cerebral cortex, hippocampus and cerebellum of rats during 6 months of treatment. The results obtained show that fish oil may be an excellent source of n-3 PUFA which incorporates into brain tissues when it is present in the diet. Experimental forage was used that contained $33.58 \%$ PUFA ( $n-3$ plus n- $6 \%$ of total fatty acids). The most abundant n-3 PUFA in the experimental diet are 18:3n-3 (ALA, alpha-linolenic acid), 22:6n-3 (DHA, docosahexaenoic acid) and 20:5n-3 (EPA, eicosapentaenoic acid). Additionally, to strengthen the PUFA features in the supplemented diet, SFA and MUFA levels were different, which was achieved by using various amounts of lard and rapeseed oil. Generally, in addition to significant changes

Table 3. Fatty acid composition (\% total fatty acids) in the examined brain regions of rats after 6 months of nutritional experiment

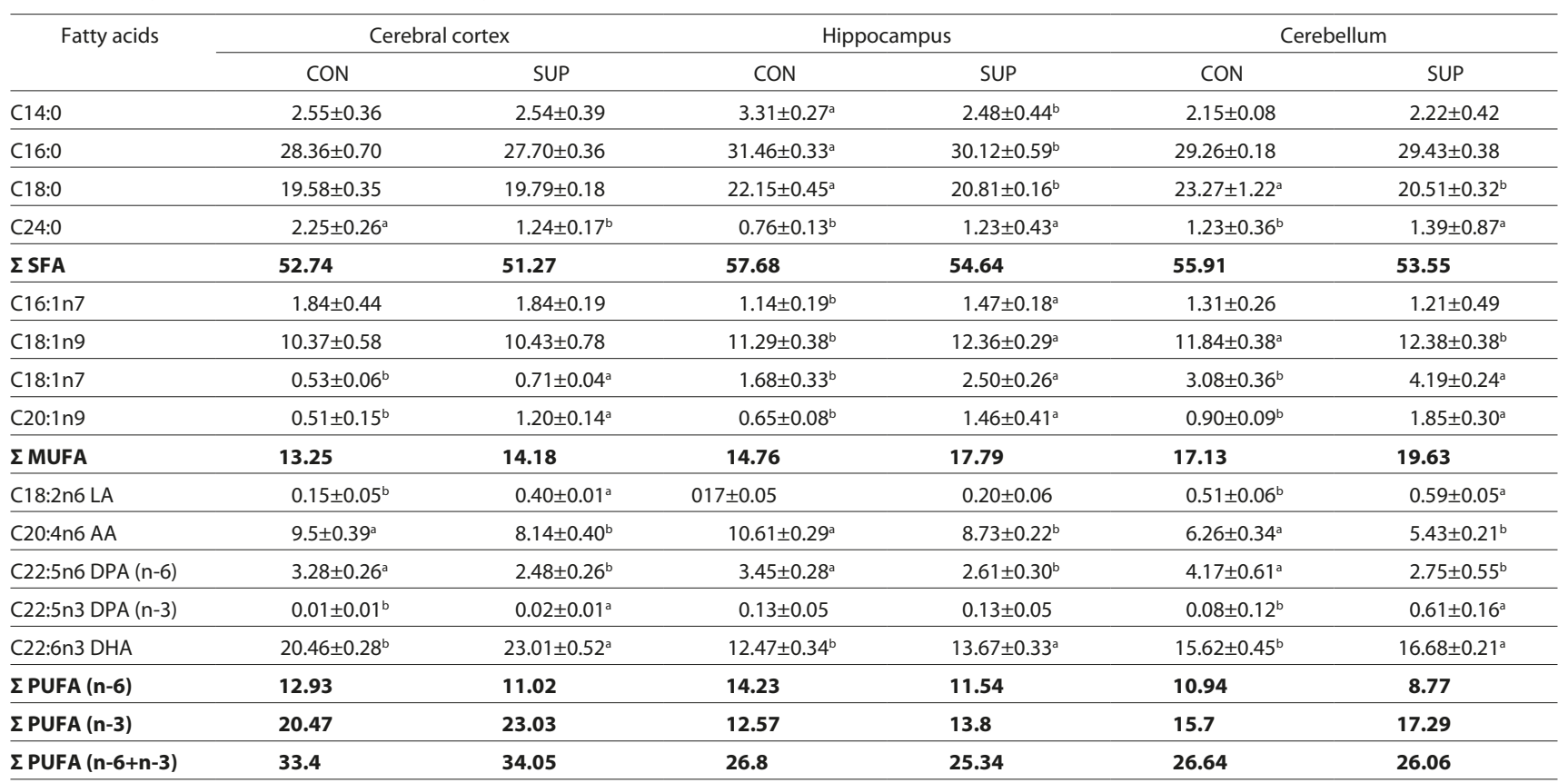

Values are mean $\pm S D$; Means with different letters $(a, b)$ are significantly different $(p \leq 0,05)$ across the same brain regions by $t$-Student test CON - control diet, SUP - supplemented diet, SFA - saturated fatty acids, MUFA - monounsaturated fatty acids, PUFA - polyunsaturated fatty acids. 
of PUFAs in different regions of the brain, changes in SFA and MUFA levels were also noted. Saturated fatty acid (SFA) content was least modified by the administered diet when compared in all tested brain regions in rat groups. Significant differences among all the fatty acids analyzed were observed only in the hippocampus in the experimental group. In the cerebral cortex, changes related only to $24: 0$, whereas in the cerebellum, it included changes to 24:0 and also16:0. In a similar study conducted by Carrie et al (2000), there was no effect of diets (n-3 deficient diet, egg yolk phospholipid diet, brain cerebral phospholipid diet) on SFA or MUFA level in the brain of mice [10]. The results obtained in the presented study, which analyzed monounsaturated fatty acids (MUFA), demonstrate a clear trend showing an increase in the levels of these fatty acids in all examined parts of the brain in the supplemented group. Most of the statistically significant changes in MUFA concentration were recorded in the hippocampus. In studies by Murthy et al (2002), there was a small decrease (from about $25 \%-20-22 \%$ ) in total $s n-1$ 18:1 species in PE-plasmalogens (PLE) in n-3 deficient rats, which is consistent with an earlier report indicating that fish oil feeding increases the proportion of PLE species containing $s n-1$ 18:1 in monkey brains [11]. The presented results also demonstrated that PUFA content in rat brain was modified by diet. Dietary fish oil supplementation demonstrates that 22:6n-3 incorporated effectively into all tested brain regions and reduced the level of C20:4n-6 as well as C22:5n-6. It is suggested that dietary 22:6n-3 appears to compete directly with C20:4n-6 for the desaturation systems, because n- 6 and n-3 fatty acids use the same enzymes, and desaturation is a competitive substrate and produces inhibition.

The fatty acid composition of neurons during aging has been associated with reduction in PUFA levels, progressive decline in saturated fatty acids and reciprocal increases in monounsaturated fatty acids. This can be modified to a certain degree by dietary alternations, e.g., increased intake of 20:5n-3 can significantly increase brain content of $20: 5 n-3$ and metabolite 22:5n-3 in mice, even though these are trace components of the neuronal membranes. Extended intake of 22:6n-3 will modestly enhance brain DHA content, with a mutual decline in brain n-6 level, in particular 22:5n-6. The dietary deprivation of n-3 PUFA led to reduction of the brain's 22:6n-3, but with a reciprocal increase in the n- 6 level, in particular 22:5n-6. In this manner, the degree of unsaturation is more or less retained, but features of these fatty acids may be significantly different. For example, high levels of DPA, in place of DHA, leads to cognitive impairment, even if these molecules differ by only one double bond.

Infancy and old age are the 2 critical periods in which the availability of the PUFA diet plays a significant role in the proper functioning of the brain. In those periods, deficiency is associated with learning and memory deficits, sensory systems and mood. In addition, the lack of PUFAs in the diet of juveniles delays and has irreversible effects on the brain functioning of adults. Studies show that aging causes a reduction in the levels of DHA and other PUFAs in the brain due to a reduction in the capacity of those fatty acids to cross the blood-brain-barrier, and the reduction of delta-6-desaturase, less food intake, absorption difficulties, or reduced capacity to convert shorter chained fatty acids to longer fatty acids [3]. Also, metabolism changes with age: the increase in oxidative stress results in a lower concentration of $n-3$ in the nervous system.
The presented studies demonstrates that long-term administration of fish oil rich in long chain fatty acids affects the composition of fatty acids in various brain areas, especially regarding the increase in DHA. In the face of the significant age-dependent decrease in the levels and turnover of the PUFA in the hippocampus, the cortex, striatum and hypothalamus, [12] these results suggest that a diet rich in n-3 PUFA can restore an adequate level of fatty acid in the aging brain.

\section{CONCLUSIONS}

In summary, the above-data strongly suggest that dietetic fish oil supplementation is a powerful modulator of the fatty acid turnover in the cerebral cortex, the hippocampus and cerebellum of rat brains. The 6 -month treatment of the rats with a diet enriched with fish fat increased the n-3 PUFA and decreased the n-6 PUFA levels in all tested brain regions. Furthermore, the fish oil diet modulates to a lesser extent the content of SFA and MUFA in the examined brain. It can be assumed that a diet rich in that kind of PUFAs might help reduce age-related impairments.

\section{Acknowledgement}

This work was supported by Grant No. NN312337939 from the Ministry of Science and Higher Education in Warsaw, Poland.

\section{REFERENCES}

1. Bang HO, Dyerberg J, Sinclair HM. The composition of the Eskimo food in north western Greenland. Am J Clin Nutr. 1980; 33 (12): 2657-2661.

2. Bauer JE. Therapeutic use of fish oils in companion animals. J Am Vet Med Assoc. 2011; 239 (11): 1441-1451.

3. Cole GM, Frautschy SA. DHA May Prevent Age-Related Dementia. J Nutr. 2010; 140 (4): 869-874.

4. Firlag M, Kamaszewski M, Gaca K, Adamek D, Bałasińska B. The neuroprotective effect of long term n-3 polyunsaturated fatty acids supplementation in the cerebral cortex and hippocampus of aging rats. Folia Neuropathol. 2013; 51 (3): 235-242.

5. Birch EE, Garfield S, Hoffman DR, Uauy R, Birch DG. A randomized controlled trial of early dietary supply of long-chain polyunsaturated fatty acids and mental development in term infants. Dev Med Child Neurol. 2000; 42 (3): 174-181.

6. Bousquet M, Saint-Pierre M, Julien C, Salem NJr, Cicchetti F, Calon F. Beneficial effects of dietary omega-3 polyunsaturated fatty acid on toxin-induced neuronal degeneration in an animal model of Parkinson's disease. FASEB J. 2008; 22 (4): 1213-1225.

7. Calon F, Lim GP, Morihara T, Yang F, Ubeda O, Salem NJr, Frautschy SA, Cole GM. Dietary n-3 polyunsaturated fatty acid depletion activates caspases and decreases NMDA receptors in the brain of a transgenic mouse model of Alzheimer's disease. Eur J Neurosci. 2005; 22 (3):17-26.

8. Woodruff-Pak DS, Foy MR, Akopian GG, Lee KH, Zach J, Nguyen KP, Comalli DM, Kennard JA, Agelan A, Thompson RF. Differential effects and rates of normal aging in cerebellum and hippocampus. Proc Natl Acad Sci U S A. 2010; 107 (4):1624-1629.

9. Folch J, Lees M, Sloane Stanley GH. A simple method for the isolation and purification od total lipides from animal tissues. J Biol Chem. 1957; 226 (1): 497-509.

10. Carrie I, Clément M, de Javel D, Francès H, Bourre JM. Specific phospholipid fatty acid composition of brain regions in mice: effects of n-3 polyunsaturated fatty acid deficiency and phospholipid supplementation. J Lipid Res. 2000; 41: 465-472.

11. Murthy M, Hamilton J, Greiner RS, Moriguchi T, Salem NJr, Kim HY. Differential effects of n-3 fatty acid deficiency on phospholipid molecular species composition in the rat hippocampus. J Lipid Res. 2002; 43 (4): 611-617.

12. Ledesma DM, Martin GM, Dotti CG. Lipid changes in the aged brain: Effecton synaptic function and neuronal survival. Progre Lipid Res. 2012; 51 (1): 23-35. 